\title{
Effect of 'Garbhpal Ras’ on Pregnancy Induced Nausea \& Vomiting (NVP)
}

\section{Research article}

\section{Mishra Deepa $^{1^{*}, \text { Sinha Mukta }}{ }^{2}$, Kumar Vikas $^{3}$}

1. Lecturer and In-charge, Department of Prasuti Tantra, A \& U Tibbia College and Hospital, Karol bagh, New Delhi-5.

2. Professor and Head, Department of Prasuti Tantra, Faculty of Ayurveda, Institute of

Medical Sciences, BHU, Varanasi-5.

3. Associate Professor in Pharmacology, Department of Pharmaceutics, Institute of Technology, BHU, Varanasi-5.

\begin{abstract}
Great maestros of Ayrurveda described in detail about pregnant woman and its care. During this nine month long journey, pregnant women may suffer from some minor ailments which are specific to pregnant state. Nausea and vomiting are the commonest symptoms experienced in the first trimester of pregnancy and can occur at any time of the day and may be constant. It's no wonder that child bearing women feel the desire for a remedy to overcome with these. Since ancient times, 'Garbhpal Ras' have been used for many centuries in assisting for securely child bearing process. According to author of 'Ras Chandanshu' the text of $17^{\text {th }}$ century, drug 'Garbhpal Ras' cures all minor ailments of pregnant women. 94 pregnant women were subjected for this study. Fifty five women were supplemented with $120 \mathrm{mg}$ bid 'Garbhpal Ras' along with folic acid, while 39 pregnant women of control group supplemented with folic acid only. Aim of study was to evaluate efficacy of Garbhpal Ras in nausea and vomiting during pregnancy. Result was assessed through subjective parameters (based on patient's statement), haematological and biochemical blood parameters. Results were found encouraging 'Garbhpal Ras' treated group.
\end{abstract}

Key words: Ayurveda, Garbhpal Ras, Garbhopadravas, Nausea, Vomiting, Pregnancy.

\section{Introduction:}

Pregnancy is a special time in a woman's life; from the moment of conception, the growing baby is entirely dependent on its mother. Pregnancy is a time of physical and hormonal changes and of emotional and psychological preparation for motherhood. Ayurveda has

*Corresponding Author:

\section{Mishra Deepa,}

Lecturer and In-charge,

Department of Prasuti Tantra,

A \& U Tibbia College and Hospital,

Karol bagh, New Delhi - 5 .

Email id- deepamishra219@gmail.com

Ph. no- 09650453095 suggested a very good protocol for obstetric care. It also describes certain diseases, which are due to the pregnant status of the woman. These diseases are peculiar to pregnancy and are called 'garbhopadravas', they are nausea, anorexia, vomiting, fever, oedema, anaemia, diarrhea(1). 'Kashyap' emphasized that proper management of disorders during pregnancy is helpful protection and development of both mother and fetus(2). Nausea and vomiting is a common symptom of pregnancy, affecting $70-85 \%$ of women. Mild nausea experienced during pregnancy can be normal, and should not be considered an immediate cause for alarm. There is no 
evidence of fetal damage as a result of the nausea and vomiting(3) (www.patient.co.uk). Nausea and vomiting of pregnancy is associated with favourable pregnancy outcomes, such as decreased risk of miscarriage and a lower incidence of perinatal death, low infant birth weight and preterm birth(4). However, in severe cases (Hyperemesis gravidarum) there may be an increased risk of low birth weight, congenital malformations, undescended testicles and hip dysplasia(4).

According to author of 'Ras Chandanshu' the text of $17^{\text {th }}$ century, drug 'Garbhpal Ras' cures all minor ailments of pregnant women(5,6,7). 'Garbhpal Ras' contains minerals like 'Hingula' (Cinnabar Hgs), 'Nag' (lead Pb), 'Vang' (Tin Sn) and 'Loh bhasma' (Iron Fe). Herbal contents of 'Garbhpal Ras' include 'Dalchini' (Cinnamomum zeylanicum), 'Ela' (Elettaria cardmomum), 'Tejpatra' (Cinnamomum tamala), 'Shunthi' (Zingiber officinale), 'Marich' (Piper nigrum), 'Dhanyak' (Coriandrum sativum), 'Chavya' (Piper retrofractum), 'Krishna jeerak' (Carum bulbocastanum), 'Draksha' (Vitis vinifera) and 'Devdaru' (Cedrus deodara). All ingredients in same quantity except 'Loh bhasma' (half quantity then others) were triturated in extract of 'Vishnukranta' (Clitoria ternatea).

\section{Aim of study:}

The aim of present study is to assess the efficacy of 'Garbhpal Ras' on pregnancy induced nausea and vomiting (NVP).

Prior to assess the efficacy on pregnancy induced nausea and vomiting, a thorough study was needed, so 'Garbhpal Ras' was evaluated for its acute(8), subchronic8 and chronic toxicity(9). It was also evaluated for teratogenic effects, if any, in pregnant rats and was found safe(10).

\section{Materials and methods: \\ Inclusion Criteria:}

Women belonging to age group 18 to 38 years, having amenorrhoea due to pregnancy (as soon as pregnancy detected to two month of amenorrhoea), history of spontaneous abortion, history of intra uterine death/still birth, history of preterm delivery or any other obstetric problem during previous pregnancies were included in this study.

\section{Exclusion Criteria:}

Women having essential hypertension, severe aneamia, severe degree of pregnancy induced hypertension, diabetes mellitus, hypo/ hyperthyroidism, liver or renal disorder were excluded.

\section{Criterion of selection of drug:}

The drug has been selected on the basis of authentic reference(5). 'Garbhpal Ras', a panacea for pregnancy induced minor ailments. It ensures well being of mother and fetus, as mentioned in 'Ras Chandanshu'.

\section{Grouping of patients:}

Ninty four pregnant women were randomly selected and registered from Out Patient Department, Prasuti Tantra, Sir Sunderlal Hospital, BHU, Varanasi. At the time of registration a written consent for becoming a volunteer of this study was taken from each pregnant woman.

All the registered cases have been categorized as follows

Group 1 Patients registered from first trimester having normal course of pregnancy with no specific obstetric or medical history.

Group 2 Patients registered from first trimester with risk factors like history of spontaneous abortion, pregnancy induced hypertension in previous pregnancy, preterm delivery, previous caesarean section within two years, elderly primigravida, $\quad$ TORCH (Toxoplasmosis, Rubella, 
Cytomegalovirus, Herpes) infection and on anti-tubercular treatment.

Each group is further subdivided into control and trial:

Control group $\left(\mathbf{C}_{\mathbf{1}}, \mathbf{C}_{2}\right)$ : Patient received routine medication folic acid $5 \mathrm{mg} /$ day and B-complex as advised in 'Prasuti' OPD. Combination of pyridoxine and doxylamine succinate advised to patients as per need.

Trial group $\left(\mathbf{T}_{1}, \quad \mathbf{T}_{2}\right)$ : $\quad$ Patient supplemented 'Garbhpal Ras' $120 \mathrm{mg}$ bid with milk and 3-4 'munakka' (dried form of Vitis vinifera) as anupan and folic acid $5 \mathrm{mg} /$ day.

Criteria of scoring and assessment (Table No.1)

Table 1. Criteria of scoring.

\begin{tabular}{|c|c|}
\hline Scoring & $\begin{array}{c}\text { Intensity of particular } \\
\text { symptoms }\end{array}$ \\
\hline 0 & Nil \\
\hline 1 & Mild \\
\hline 2 & Moderate \\
\hline 3 & Severe \\
\hline
\end{tabular}

\section{Parameter studied:}

Effect of Garbhpal Ras has been assessed on pregnant women considering the following-

1. Pregnancy induced nausea and vomiting.

2. Biochemical and haematological parameters (Haemoglobin, TLC, DLC, Fasting blood sugar, Blood urea, Serum creatinine, Serum bilirubin, SGOT, SGPT, Alkaline phosphatase, Serum protein, Serum albumin), Urine (routine and microscopic) and Ultrasonography (any gross congenital anomaly).

Patients were followed up at 15 days interval for 3 follow ups from second months onwards. Heamatological and biochemical tests were advised to patients before treatment and at $9^{\text {th }}$ month of gestation.

\section{Statistical analysis:}

All data were expressed in mean \pm SD. Symptoms were compared with chisquare test. Significant changes were observed in haematological and biochemical parameter through paired and unpaired ' $\mathrm{t}$ ' test.

\section{Result and discussion:}

Results have been assessed mainly on the basis of absence/ less incidence or relief in minor ailments (symptoms), untoward effects on haematological and biochemical blood parameters, if any.

Effect of Garbhpal Ras in nausea and vomiting: Table (2) shows comparison of patients suffering from nausea in trial and control group. Initially symptom of nausea found in $69.23 \%$ pregnant women control group while the occurrence was found in $67.23 \%$ women of trial group. But the severity of symptom was more (5.13\% women had severe degree of nausea) in control group. During second follow up less number of cases of nausea were observed in 'Garbhpal Ras' administered pregnant women in comparison to control, changes were significant in respect to control. This symptom disappears mostly after 3 month of pregnancy on its own. In $3^{\text {rd }}$ follow up nausea remained only in $3.62 \%$ women in trial group comparable to control $(15.38 \%)$.

Table (3) shows that initially $60 \%$ and $58.97 \%$ women of trial and control group respectively were suffered from vomiting. Severities of symptoms were more in control group $(7.96 \%$ cases of severe degree of vomiting in control and $1.82 \%$ in trial). In follow up period it was clearly seen that relief in vomiting was more in trail group, none women of 'Garbhpal Ras' group had vomiting till $3^{\text {rd }}$ follow up. Statistically significant difference was observed, while comparing $3^{\text {rd }}$ follow up with initial finding in trial group. 
Chief dosha involved in vaman is vayu, mainly the udan and vyan fractions of vayu. We know that drugs exhibit their action by dravya prabhav, guna prabhav or both dravya guna prabhav. Ela, Dalchini and Tejpatra being anulomak turned urdhvagami vata in downwards direction and thus helps to check the vaman. This action may be attributed to dravyaprabhav. Draksha, Dhanyak, Pippali, Shunthi, Ela, Tejpatra, Dalchini being madhur, pacify vata and pitta both so relieves vaman by gunaprabhav.

Ayurvedic sources notify that ginger is the best remedy for morning sickness in pregnancy(11). There is a sufficient amount of researches data to show that ginger (Shunthi) play an effective role against pregnancy related morning sickness $(12,13,14,15)$. It may be the possible reason of improvement in symptoms of nausea and vomiting in Garbhpal Ras administered group. Dhanyak (11) and Ela(16) have also shown similar effect.

\section{Effect of Garbhpal Ras on heamatological parameters:}

The level of haemoglobin was found significantly increased in pregnant women administered with 'Garbhpal Ras' as well as in cases of control group. Initially, in $\mathrm{T}_{1}, \mathrm{~T}_{2}$ group haemoglobin level was $10.25,10.32 \mathrm{gm} / \mathrm{dl}$ respectively. In last month these were improved to become $12.14,12.20 \mathrm{gm} / \mathrm{dl}$. Although every patient was supplemented with iron preparation, increase in haemoglobin percentage was found statistically significant only in pregnant women taking 'Garbhpal Ras' from $1^{\text {st }}$ trimester and belong to risk group $\left(\mathrm{T}_{2}\right)$ with respective control (Table 4$)$. It shows that 'Garbhpal Ras' causes no interference/ impairment in absorption of iron rather it might have synergistic effect.

It was observed that average mean value of total leukocytes count was increased significantly in last month, though it was a physiological change and was within normal limits. Changes in the hematological parameters of the trial group were found statistically not significant in comparison to control. It shows that 'Garbhpal Ras' does not produce any haemopoitic toxicity (Table 4).

\section{Effect of Garbhpal Ras on biochemical parameters of blood:}

Level of blood sugar was found within normal range in all groups. No incidence of hypo/ hyperglycemia was observed in any of the case (Table 4). Liver function (serum bilirubin, SGOT, SGPT and alkaline phosphatase) and renal function (blood urea, serum creatinine) were maintained during ante-natal period in trial group as well as control group (Table 5 and 6). Normal levels of biochemical parameters till the end of treatment, show non-toxic nature of 'Garbhpal Ras'.

\section{Conclusion:}

It is difficult to completely prevent pregnancy induced nausea and vomiting, as it is a natural and physiological phenomenon. However from above data it seems that 'Garbhpal Ras' prevent nausea and vomiting to become a major problem and it also limits the periods of nausea and vomiting in present study. Improve hemoglobin level and normal biochemical and haematological parameters show non toxic nature of drug. Therefore, knowledge of the findings can be applied for nursing practice to improve maternal well being.

\section{Acknowledgement:}

Authors are thankful to Dabur India Ltd., Sahibabad, UP for providing gift sample of 'Garbhpal Ras'. Deepa Mishra is thankful to the University Grants Commission, New Delhi for providing Junior Research Fellowship. 


\section{References:}

1. Harit. Edited and Hindi commentary by Pt. Ramavalamb Shastri. Harit Samhita. 1ed. Varanasi. Prachhya Prakashan . 1985. 400p.

2. Kashyap. Text with English translation and Commentary by P.V. Tewari. Kashyap Samhita.Varanasi. Chaukhambha Visvabharati. 2002. 553p.

3. Available on: http://www.patient.co.uk/showdoc/400 24911.

4. Weigel RM. and Weigel MM. Nausea and vomiting of early pregnancy and pregnancy outcome. A meta-analytical review. Br J Obstet Gynaecol. 1989; 96(11);1312-1318.

5. Duttaram Vaidya. Translated by Sri Hari Prapanna Sharma. Rasyog Sagar. Krishna Das Academy. Vol.I, 2004. 374-375p.

6. Chhangadi GS. Ras Tantra Sar va Sidhdhi Prayog Sangrah. Vol. I, 9 ed. Ajmer. Krishna Gopal Ayurveda Bhavan. 1999. 554-555p.

7. Goyal RK. and Mahajan, R. Adhyatan Ras shastra. 1 ed. Varanasi. Chuakhambha Surbharati Prakashan. 1988. 309p.

8. Mishra D., Sinha M., Singh P.N. and Kumar V. Acute and sub-chronic toxicity study of Garbhpal Ras. Electronic J Pharmacol Therapy. 2008; $1 ; 31-34$.
9. Mishra D., Sinha M., Kumar M. and Kumar V. Chronic Toxicity Study of 'Garbhpal Ras'; An Ayurvedic Medicine. J Herb Med Toxicol. 2009; 3(1); 13-17.

10. Mishra D., Sinha M. and Kumar V. Teratological Study of 'Garbhpal Ras', An Ayurvedic Formulation. J Herb Med Toxicol. 2009; 3(1); 37- 40.

11. Brahma Shankar Shastri. Vidyotini hindi Commentry by Lakshmi Pati Shastri. Yoga Ratanakara. 1ed. Varanasi. Chaukhambha Sanskrit Sansthan. 1995. 421p.

12. Borrelli F., Capasso R., Aviello G., Pittler MH. and Izzo AA. Effectiveness and safety of ginger in the treatment of pregnancy induced nausea and vomiting. Obstet Gynaecol. 2005; 105; 849-856.

13. Mowrey DB. and Clayson DE. Motion sickness, Ginger and psychophysics. Lancet.1982; 1; 655-657.

14. Jewell D. and Young G. Interventions for nausea and vomiting in early pregnancy. Cochrane database syst Rev. (4): CD000145, 2003.

15. Available on: http://www.umm.edu/altmed/ConsHer bs/Gingerch.html. 2006.

16. Mishra D. and Neelam. Effect of Laja and Chaturjatak choorna in Garbhaj Vaman. The Antiseptic. 2007; 104(8); 428-430. 
Table 2. Effect of 'Garbhpal Ras' on nausea.

\begin{tabular}{|c|c|c|c|c|c|c|c|c|c|c|}
\hline \multirow[t]{3}{*}{ Group } & \multirow[t]{3}{*}{ Score } & \multicolumn{8}{|c|}{ Follow Up* } & \multirow{3}{*}{$\begin{array}{l}\text { Initial Vs } \\
\text { III }\end{array}$} \\
\hline & & \multicolumn{2}{|c|}{ Initial } & \multicolumn{2}{|l|}{$\mathrm{I}$} & \multicolumn{2}{|l|}{ II } & \multicolumn{2}{|l|}{ III } & \\
\hline & & No. & $\%$ & No. & $\%$ & No. & $\%$ & No. & $\%$ & \\
\hline \multirow{4}{*}{$\begin{array}{l}\mathrm{T}=\mathrm{T}_{1}+\mathrm{T} 2 \\
(\mathrm{n}=55)\end{array}$} & 0 & 18 & 32.73 & 12 & 21.81 & 39 & 70.91 & 53 & 96.36 & \multirow{5}{*}{$\begin{array}{l}\chi^{2}=14.565 \\
P<0.01 S\end{array}$} \\
\hline & 1 & 29 & 52.73 & 32 & 58.18 & 16 & 29.10 & 2 & 3.64 & \\
\hline & 2 & 7 & 12.73 & 11 & 20 & 0 & 0 & 0 & 0 & \\
\hline & 3 & 1 & 1.82 & 0 & 0 & 0 & 0 & 0 & 0 & \\
\hline \multicolumn{2}{|l|}{ Mean \pm SD } & \multicolumn{2}{|c|}{$0.84 \pm 0.71$} & \multicolumn{2}{|c|}{$0.98 \pm 0.65$} & \multicolumn{2}{|c|}{$0.29 \pm 0.46$} & \multicolumn{2}{|c|}{$0.04 \pm 0.19$} & \\
\hline \multirow{4}{*}{$\begin{array}{l}C=C_{1}+C_{2} \\
(n=39)\end{array}$} & 0 & 12 & 30.77 & 5 & 12.82 & 23 & 58.97 & 33 & 84.62 & \multirow{4}{*}{$\begin{array}{l}\chi^{2}=5.183 \\
P<0.05 \quad S\end{array}$} \\
\hline & 1 & 19 & 48.72 & 15 & 38.46 & 14 & 35.90 & 5 & 12.82 & \\
\hline & 2 & 6 & 15.38 & 16 & 41.03 & 2 & 5.13 & 1 & 2.56 & \\
\hline & 3 & 2 & 5.13 & 3 & 7.69 & 0 & 0 & 0 & 0 & \\
\hline \multicolumn{2}{|l|}{ Mean \pm SD } & \multicolumn{2}{|c|}{$0.95 \pm 0.83$} & \multicolumn{2}{|c|}{$1.44 \pm 0.82$} & \multicolumn{2}{|c|}{$0.46 \pm 0.60$} & \multicolumn{2}{|c|}{$0.18 \pm 0.45$} & \\
\hline \multicolumn{2}{|c|}{$\begin{array}{l}\text { Intergroup } \\
\text { Comparison with } \\
\text { Chi- square test }\end{array}$} & \multicolumn{2}{|c|}{$\begin{array}{l}\chi^{2}=0.577 \\
P>0.05 \quad \mathrm{NS}\end{array}$} & \multicolumn{2}{|c|}{$\begin{array}{l}\chi^{2}=8.693 \\
P<0.001 \\
\text { HS }\end{array}$} & \multicolumn{2}{|c|}{$\begin{array}{l}\chi^{2}=0.965 \\
P>0.05 \quad \mathrm{NS}\end{array}$} & \multicolumn{2}{|c|}{$\S$} & \\
\hline
\end{tabular}

*at very 15 days interval

$\S$ Frequency of column is less than 5, so can't be compared.

Table 3. Effect of 'Garbhpal Ras' on vomiting.

\begin{tabular}{|c|c|c|c|c|c|c|c|c|c|c|}
\hline \multirow{3}{*}{ Group } & \multirow{3}{*}{ Score } & \multicolumn{8}{|c|}{ Follow Up* } & \multirow{3}{*}{$\begin{array}{c}\text { Initial Vs } \\
\text { III }\end{array}$} \\
\hline & & \multicolumn{2}{|c|}{ Initial } & \multicolumn{2}{|c|}{ I } & \multicolumn{2}{|c|}{ II } & \multicolumn{2}{|c|}{ III } & \\
\hline & & No. & $\%$ & No. & $\%$ & No. & $\%$ & No. & $\%$ & \\
\hline \multirow{4}{*}{$\begin{array}{c}\mathrm{T} \\
=\mathrm{T}_{1}+\mathrm{T} 2 \\
(\mathrm{n}=55)\end{array}$} & 0 & 22 & 40 & 14 & 25.45 & 37 & 67.27 & 55 & 100 & \multirow{5}{*}{$\begin{array}{c}\chi^{2}=7.165 \\
\mathrm{P}<0.01 \\
\text { HS }\end{array}$} \\
\hline & 1 & 24 & 43.63 & 29 & 52.73 & 18 & 32.73 & 0 & 0 & \\
\hline & 2 & 8 & 14.55 & 12 & 21.82 & 0 & 0 & 0 & 0 & \\
\hline & 3 & 1 & 1.82 & 0 & 0 & 0 & 0 & 0 & 0 & \\
\hline \multicolumn{2}{|c|}{ Mean \pm SD } & \multicolumn{2}{|c|}{$0.78 \pm 0.76$} & \multicolumn{2}{|c|}{$0.96 \pm 0.69$} & \multicolumn{2}{|c|}{$0.33 \pm 0.47$} & \multicolumn{2}{|c|}{$0.00 \pm 0.00$} & \\
\hline \multirow{4}{*}{$\begin{array}{c}C \\
=C_{1}+C_{2} \\
(n=39)\end{array}$} & 0 & 16 & 41.03 & 5 & 12.80 & 22 & 56.41 & 34 & 87.18 & \multirow{5}{*}{$\begin{array}{c}\chi^{2}=1.524 \\
P>0.05 \\
\quad N S\end{array}$} \\
\hline & 1 & 15 & 38.46 & 13 & 33.33 & 15 & 38.46 & 5 & 12.82 & \\
\hline & 2 & 5 & 12.80 & 18 & 46.13 & 2 & 5.13 & 0 & 0 & \\
\hline & 3 & 3 & 7.69 & 3 & 7.69 & 0 & 0 & 0 & 0 & \\
\hline \multicolumn{2}{|c|}{ Mean \pm SD } & \multicolumn{2}{|c|}{$0.87 \pm 0.92$} & \multicolumn{2}{|c|}{$1.49 \pm 0.82$} & \multicolumn{2}{|c|}{$0.49 \pm 0.60$} & \multicolumn{2}{|c|}{$0.13 \pm 0.34$} & \\
\hline \multicolumn{2}{|c|}{$\begin{array}{c}\text { Intergroup } \\
\text { Comparison with } \\
\text { Chi- square test }\end{array}$} & \multicolumn{2}{|c|}{$\begin{array}{c}\chi^{2}=0.259 \\
P>0.05 \\
\text { NS }\end{array}$} & \multicolumn{2}{|c|}{$\begin{array}{c}\chi^{2}=10.391 \\
\mathrm{P}>0.01 \\
\text { HS }\end{array}$} & \multicolumn{2}{|c|}{$\begin{array}{c}\chi^{2}=0.734 \\
P>0.05 \\
\quad N S\end{array}$} & \multicolumn{2}{|c|}{$\S$} & \\
\hline
\end{tabular}

*at every 15 days interval

$\S$ Frequency of column is less than 5, so can't be compared. 
Table 4. Effect of 'Garbhpal Ras' on haematology parameters and blood sugar level.

\begin{tabular}{|c|c|c|c|c|c|c|}
\hline \multirow{2}{*}{ Group } & \multicolumn{2}{|c|}{ Haemoglobin $(\mathrm{gm} \%)$} & \multicolumn{2}{|c|}{ TLC $\left(\mathrm{mm}^{3}\right)$} & \multicolumn{2}{c|}{ FBS (mg/dl) } \\
\cline { 2 - 7 } & BT & AT & BT & AT & BT & AT \\
\hline T1 & 10.25 & 12.14 & 8347.22 & 11244.44 & 72.04 & 73.25 \\
$(\mathrm{n}=30)$ & \pm 1.22 & $\pm 1.10 \dagger \dagger$ & \pm 1103.37 & $\pm 1162.87 \dagger \dagger$ & \pm 12.98 & $\pm 10.51^{*}$ \\
\hline C1 & 10.23 & 11.58 & 8652.15 & 11808.71 & 78.76 & 79.42 \\
$(\mathrm{n}=20)$ & \pm 1.23 & $\pm 1.10 \dagger \dagger$ & \pm 1192.18 & $\pm 1240.20 \dagger \dagger$ & \pm 11.98 & \pm 10.58 \\
\hline T2 & 10.32 & 12.20 & 9373.33 & 11295.65 & 76.06 & 76.29 \\
$(\mathrm{n}=25)$ & \pm 1.63 & $\pm 0.92 \dagger \dagger^{*}$ & \pm 1038.03 & $\pm 1157.38 \dagger \dagger$ & \pm 11.97 & \pm 9.38 \\
\hline $\mathrm{C} 2$ & 10.50 & 11.81 & 9121.08 & 11989.47 & 75.05 & 77.93 \\
$(\mathrm{n}=19)$ & \pm 1.39 & $\pm 0.88 \dagger$ & \pm 1003.10 & $\pm 1191.12 \dagger \dagger$ & \pm 11.29 & \pm 10.64 \\
\hline
\end{tabular}

${ }^{*} \mathrm{p}<0.01$, compared to control; $\uparrow \mathrm{p}<0.01$, compared to BT; $\uparrow \dagger \mathrm{p}<0.001$, compared to BT.

Table 5. Effect of 'Garbhpal Ras' on renal function.

\begin{tabular}{|c|c|c|c|c|}
\hline \multirow{2}{*}{ Group } & \multicolumn{2}{|c|}{ Blood Urea $(\mathrm{mg} / \mathrm{dl})$} & \multicolumn{2}{c|}{ Serum Creatinine $(\mathrm{mg} / \mathrm{dl})$} \\
\cline { 2 - 5 } & BT & AT & BT & AT \\
\hline T1 $(n=30)$ & $16.29 \pm 3.08$ & $16.76 \pm 2.12$ & $0.65 \pm 0.08$ & $0.70 \pm 0.08$ \\
\hline C1 $(n=20)$ & $17.20 \pm 3.20$ & $17.9 \pm 2.32 \uparrow$ & $0.66 \pm 0.13$ & $0.67 \pm 0.09$ \\
\hline T2 $(n=25)$ & $16.36 \pm 2.25$ & $16.33 \pm 1.90$ & $0.68 \pm 0.12$ & $0.72 \pm 0.09$ \\
\hline C2 $(n=19)$ & $17.98 \pm 3.32$ & $16.77 \pm 3.50$ & $0.69 \pm 0.13$ & $0.71 \pm 0.10$ \\
\hline
\end{tabular}

${ }^{*} \mathrm{p}<0.01$, compared to control; $\uparrow \mathrm{p}<0.01$, compared to BT.

Table 6. Effect of 'Garbhpal Ras' on liver function test.

\begin{tabular}{|c|c|c|c|c|c|c|c|c|c|c|c|c|}
\hline \multirow{2}{*}{$\begin{array}{c}\text { Grou } \\
\mathrm{p}\end{array}$} & \multicolumn{2}{|c|}{$\begin{array}{l}\text { Serum } \\
\text { Bilirubin } \\
(\mathrm{mg} / \mathrm{dl})\end{array}$} & \multicolumn{2}{|c|}{$\begin{array}{l}\text { SGOT } \\
(\mathrm{U} / \mathrm{ml})\end{array}$} & \multicolumn{2}{|c|}{$\begin{array}{l}\text { SGPT } \\
(\mathrm{U} / \mathrm{ml})\end{array}$} & \multicolumn{2}{|c|}{$\begin{array}{c}\text { Alkaline } \\
\text { Phosphatase } \\
\text { (U) }\end{array}$} & \multicolumn{2}{|c|}{$\begin{array}{l}\text { Serum } \\
\text { Protien } \\
(\mathrm{gm} / \mathrm{dl})\end{array}$} & \multicolumn{2}{|c|}{$\begin{array}{l}\text { Serum } \\
\text { Albumin } \\
(\mathrm{gm} / \mathrm{dl})\end{array}$} \\
\hline & BT & $\mathrm{AT}$ & BT & AT & BT & AT & BT & $\mathrm{AT}$ & $\mathrm{BT}$ & $\mathrm{AT}$ & $\mathrm{AT}$ & BT \\
\hline $\begin{array}{c}\mathrm{T} 1 \\
(\mathrm{n}=3 \\
0)\end{array}$ & $\begin{array}{c}0.64 \\
\pm 0.1 \\
2\end{array}$ & $\begin{array}{c}0.60 \\
\pm 0.1 \\
1\end{array}$ & $\begin{array}{c}28.0 \\
3 \\
+4.6 \\
7\end{array}$ & $\begin{array}{c}27.56 \\
\pm \\
4.40\end{array}$ & $\begin{array}{c}35.1 \\
2 \\
\pm 4.7 \\
8\end{array}$ & $\begin{array}{c}35.3 \\
2 \\
\pm 4.6 \\
5\end{array}$ & $\begin{array}{c}142.7 \\
7 \\
\pm 24.3 \\
4\end{array}$ & $\begin{array}{c}149.8 \\
0 \\
\pm 23.5 \\
0 \dagger\end{array}$ & $\begin{array}{c}6.48 \\
\pm 0.5 \\
8\end{array}$ & $\begin{array}{c}6.44 \\
\pm 0.5 \\
0\end{array}$ & $\begin{array}{c}3.59 \\
\pm 0.4 \\
0\end{array}$ & $\begin{array}{c}3.56 \\
\pm 0.4 \\
1\end{array}$ \\
\hline $\begin{array}{c}\mathrm{C} 1 \\
(\mathrm{n}=2 \\
0)\end{array}$ & $\begin{array}{c}0.68 \\
\pm 0.0 \\
8\end{array}$ & $\begin{array}{c}0.56 \\
\pm 0.1 \\
1\end{array}$ & $\begin{array}{c}29.0 \\
1 \\
+5.8 \\
8\end{array}$ & $\begin{array}{l}29.10 \\
\pm 4.94\end{array}$ & $\begin{array}{c}36.0 \\
5 \\
\pm 6.3 \\
9\end{array}$ & $\begin{array}{c}33.9 \\
0 \\
\pm 5.0 \\
6\end{array}$ & $\begin{array}{c}154.4 \\
5 \\
\pm 25.0 \\
3\end{array}$ & $\begin{array}{c}158.9 \\
0 \pm \\
25.68\end{array}$ & $\begin{array}{c}6.67 \\
\pm 0.6 \\
0\end{array}$ & $\begin{array}{c}6.62 \\
\pm 0.5 \\
8\end{array}$ & $\begin{array}{c}3.51 \\
\pm 0.4 \\
3\end{array}$ & $\begin{array}{c}3.60 \\
\pm 0.4 \\
0\end{array}$ \\
\hline $\begin{array}{c}\mathrm{T} 2 \\
(\mathrm{n}=2 \\
5)\end{array}$ & $\begin{array}{c}0.62 \\
\pm 0.1 \\
3\end{array}$ & $\begin{array}{c}0.57 \\
\pm 0.1 \\
2\end{array}$ & $\begin{array}{c}27.7 \\
8 \\
\pm 6.2 \\
8\end{array}$ & $\begin{array}{c}27.53 \\
\pm \\
4.19\end{array}$ & $\begin{array}{c}35.4 \\
0 \\
\pm 4.3 \\
7\end{array}$ & $\begin{array}{c}35.2 \\
3 \\
\pm 4.1 \\
6\end{array}$ & $\begin{array}{c}142.4 \\
4 \\
+37.1 \\
2\end{array}$ & $\begin{array}{c}147.2 \\
2 \pm \\
32.28\end{array}$ & $\begin{array}{c}6.47 \\
\pm 0.4 \\
9\end{array}$ & $\begin{array}{c}6.58 \\
\pm 0.4 \\
3\end{array}$ & $\begin{array}{c}3.36 \\
\pm 0.4 \\
1\end{array}$ & $\begin{array}{c}3.40 \\
\pm 0.4 \\
0\end{array}$ \\
\hline $\begin{array}{c}\mathrm{C} 2 \\
(\mathrm{n}=1 \\
9)\end{array}$ & $\begin{array}{c}0.63 \\
\pm 0.1 \\
2\end{array}$ & $\begin{array}{c}0.60 \\
\pm 0.1 \\
3\end{array}$ & $\begin{array}{c}27.3 \\
2 \\
\pm 6.5 \\
7\end{array}$ & $\begin{array}{c}27.21 \\
\pm 4.21 \\
\dagger\end{array}$ & $\begin{array}{c}34.6 \\
9 \\
\pm 6.6 \\
5\end{array}$ & $\begin{array}{c}35.7 \\
9 \\
\pm 5.8 \\
0\end{array}$ & $\begin{array}{c}144.2 \\
1 \\
\pm 31.1 \\
6\end{array}$ & $\begin{array}{c}140.6 \\
3 \pm \\
34.16\end{array}$ & $\begin{array}{c}6.38 \\
\pm 0.4 \\
9\end{array}$ & $\begin{array}{c}6.60 \\
\pm 0.3 \\
6\end{array}$ & $\begin{array}{c}3.44 \\
\pm 0.3 \\
7\end{array}$ & $\begin{array}{c}3.55 \\
\pm 0.3 \\
6\end{array}$ \\
\hline
\end{tabular}

$* \mathrm{p}<0.01$, compared to control; $\uparrow \mathrm{p}<0.01$, compared to BT. 